Ágora Rev. Cient. 2018; 05(02):e6

\title{
Percepción de los egresados sobre el proceso enseñanza- aprendizaje en una Universidad Privada
}

\author{
Perception of graduates about the teaching-learning process in a Private University \\ Anika Remuzgo Artezano ${ }^{1}$, María Cárdenas de Fernández ${ }^{1,2}$
}

\section{RESUMEN}

Introducción: La Universidad tiene la responsabilidad de fortalecer el desarrollo social, económico y político del país, ella forma estudiantes con visión de liderazgo, con la finalidad que alcancen éxito y orienten su entorno laboral. Objetivo: El objetivo del estudio fue determinar la percepción de los egresados sobre el proceso enseñanza aprendizaje según dimensiones: plan curricular, estrategias de enseñanza aprendizaje, desarrollo de actividades y evaluación en una Universidad Privada. Materiales y métodos: La investigación es de tipo descriptivo, transversal y de enfoque cuantitativo, busca responder problemas relacionados con la percepción de los egresados sobre el proceso enseñanza aprendizaje según dimensiones. Resultados: En los resultados se caracterizan las dimensiones estructurados en el Factor de Enseñanza aprendizaje, fundamentados en el Marco conceptual del Modelo de SINEACE. Dimensión: Plan Curricular, la percepción que tienen los egresados en relación a la dimensión Plan Curricular es buena representando un $48.3 \%$ con tendencia a excelente. Al respecto los egresados han manifestado una posición intermedia, debido a las variaciones que ha presentado el Plan curricular, lo cual han considerado positivo para su desarrollo profesional. Conclusión: Se pudo concluir también respecto al proceso de enseñanza aprendizaje: disconformidad respecto al plan curricular, expresando que existe inadecuada secuencia de los cursos y, respecto a las estrategias de enseñanza y aprendizaje, refieren que la metodología de las sesiones fueron con predominio tradicional haciendo uso solo de multimedia.

Palabras clave: percepción, proceso enseñanza-aprendizaje, plan curricular.

\begin{abstract}
Introduction: The University has the responsibility to strengthen the social, economic and political development of the country, it forms students with a vision of leadership, in order to achieve success and guide their work environment. Objective: The objective of the study was to determine the perception of the graduates about the teaching-learning process according to dimensions: curricular plan, teaching-learning strategies, development of activities and evaluation in a Private University. Materials and methods: The research is of a descriptive, transversal and quantitative approach, seeks to answer problems related to the perception of the graduates about the teaching-learning process according to dimensions. Results: In the results, the structured dimensions in the Learning Teaching Factor are characterized, based on the Conceptual Framework of the SINEACE Model. Dimension: Curricular Plan, the perception that the graduates have in relation to the Curricular Plan dimension is good, representing $48.3 \%$ with an excellent tendency. In this regard, the graduates have manifested an intermediate position, due to the variations that the curricular Plan has presented, which they considered positive for their professional development. Conclusion: It was also possible to conclude regarding the teaching-learning process: disagreement with the curricular plan, expressing that there is an inadequate sequence of the courses and, regarding the teaching and learning strategies, they refer that the methodology of the sessions was traditionally predominant use only of multimedia.
\end{abstract}

Keywords: perception, teaching-learning process, curricular plan.

${ }^{1}$ Escuela de Enfermería. Universidad Norbert Wiener. Lima - Perú.

${ }^{2}$ Universidad de Carabobo. Venezuela. 


\section{INTRODUCCIÓN}

La Universidad es una institución que tiene la responsabilidad de fortalecer el desarrollo social, económico y político de un país, por lo que forma estudiantes con visión de liderazgo, que sean capaces de aplicar herramientas para favorecer su desenvolvimiento científico e innovador, con la finalidad de que alcancen éxito en el control de su entorno laboral. En este contexto, la Universidad es la llamada a liderar el proceso de innovación como fuente de inspiración para que sus estudiantes generen nuevos conocimientos, se enfrenten a desafíos y asuman acciones que propongan soluciones a los problemas que identifican en la sociedad del siglo XXI (1).

Ante ello los expertos afirman que actualmente la universidad, como espacio educativo, no ocupa un lugar relevante en la producción del conocimiento ni tampoco en el tratamiento de los problemas sociales. Probablemente, porque el ahogo político impacta a diario en recortes presupuestarios, con el consecuente deterioro de la infraestructura y de las en condiciones profesionales de docentes. Lo que hace que este nivel educativo sea inerte y rutinario en consecuencia no produzca cambios que ayuden a revertir la actual situación nacional.

Según Castellanos, para realmente alcanzar el objetivo de la transformación, se requiere de trabajar directa y progresivamente con el estudiante en un complejo proceso de socialización, para que asuma una posición activa y responsable en su formación profesional frente al desarrollo de actividades curriculares que se le brindan en el Proceso de Enseñanza Aprendizaje (PEA) (2). Ante ello se hace necesario reconocer que para obtener entornos favorables de aprendizaje se requiere de dimensiones de tipo organizativo, pedagógico y tecnológico (3).

Para esto es necesario que la relación profesoralumno presente algunas configuraciones que la hacen especialmente diferente de cualquier otra; interpersonal: primero porque la relación entre el profesor y el alumno no se establece sobre la base de simpatía mutua, afinidad de caracteres o de intereses comunes se funda en una cierta imposición, después porque es una relación bipolar de ida y vuelta que se establece entre personas de diferente edad y grado de madurez mental (4).

Por otro lado, se conoce que enfermería, se destacó en la década de los 80 por la consolidación de la disciplina, y ante ello las distintas corrientes profesionales trabajaron por el desarrollo de un cuerpo de conocimientos propio, que brindaran un nuevo marco de definición de la responsabilidad de las enfermeras/os en la atención a la salud de la población.

Por aquel entonces la formación de enfermería, que hasta ese momento había sido eminentemente práctica, modificó su diseño curricular replanteando el peso equivalente en la distribución de teoría y práctica clínica. Dicha visión de la realidad favoreció la tendencia de una mirada integral del currículo, con una amplia formación de conocimientos basados en las investigaciones, que trabajaran con enfoque multidisciplinario y trans-disciplinario, mostrando liderazgo en cada una de sus actuaciones.

Según Peñaloza "Un profesional integro, es capaz de integrarse a la sociedad activamente, para incorporarse a la cultura del país y recoger lo más valioso de la cultura universal...." (5). Por ello enfatiza la necesidad de las instituciones formadoras con currículo integral, para generar la oportunidad de desarrollar armónicamente conocimientos, prácticas profesionales, investigación, tutoría y cultura. Cabe destacar que el autor, resalta la integración de las dimensiones que como ser humano se posee para relacionar el entorno sociocultural con un permanente sentido crítico (6).

Por otro lado a partir del 2009, el Sistema Nacional de Evaluación, Acreditación y Certificación de Calidad de la Educación Superior Universitaria (SINEACE), acreditó en la carrera de enfermería a la Universidad Católica de los Ángeles de Chimbote, Universidad Nacional del Santa, Universidad Privada Norbert Wiener, Universidad Nacional Hermilio Valdizan, Universidad Ricardo Palma, Universidad Privada Antenor Orrego, Universidad Nacional de Trujillo, Universidad Católica Santo Toribio de Mogrovejo y Universidad Peruana Unión, con la finalidad de mejorar la calidad del PEA, gestión, investigación, extensión universitaria y proyección social (7).

$\mathrm{Al}$ respecto se conoce que en el proceso de evaluación de la calidad educativa, es considerada importante la percepción de los egresados sobre el PEA, siendo el punto de partida de toda gestión de calidad en procesos educativos. Un ejemplo de ello es el estudio de Miranda sobre "Percepción de los estudiantes acerca de la enseñanza teórico practico que reciben" donde identificaron que la percepción que tienen los estudiantes de enfermería y demás escuelas, acerca de la metodología que emplea el docente en el PEA es medianamente favorable con tendencia a desfavorable respecto a la teoría y la práctica" (8). Así mismo el estudio en el programa de segunda especialización de enfermería en una Universidad 
Pública, identificó que el PEA es medianamente favorable con tendencia al desfavorable (3). Por su parte Cestari luego de estudiar un curso de graduación de enfermeras concluye que la comunicación establecida entre estudiantes, profesores y pacientes, ejerce un rol fundamental en el desarrollo del PEA, pero ni en este estudio ni en los previos se evidenció la percepción de egresados de una universidad Privada de Lima sobre el PEA desde una perspectiva cuantitativa (9).

Es por eso que la filosofía de la educación ha ejercido una enorme influencia, así tenemos que Dewey (10). En sus escritos sobre filosofía de la educación, concibe el conocimiento humano como una transición que va desde una problemática, hasta una situación mejorada. Por lo que dicha transición implica al menos dos componentes: la reflexión y la acción. Solo o aislado cualquiera de los componentes resulta carente de sentido. La acción sin la reflexión es ciega, es decir, no tiene objeto, no ayuda al progreso. La reflexión sin la acción carece de significado, dado que el conocer es algo prospectivo, reformista, hipotético.

Con lo cual nos acercamos a lo que Lonergan caracteriza como filosofía y educación. Para este intelectual "la filosofía es un pensar fundamental acerca de la situación humana. Y la educación es el gran medio para transformar la situación humana" (11). En otras palabras, la filosofía viene a ser el componente reflexivo, y la educación es el componente activo.

Es bueno mencionar que el aprendizaje es un término que desde una perspectiva de Psicología educativa, según Woolfolk A. (12). "ocurre cuando la experiencia genera un cambio relativamente permanente en los conocimientos o las conductas de un individuo. El cambio puede ser deliberado o involuntario, para mejorar o empeorar, correcto o incorrecto, y consciente o inconsciente", así que esto permite decir que dentro del aprendizaje los cambios resultantes de este proceso influyen posteriormente en los conocimientos o en la conducta de los individuos.

De tal forma que el aprendizaje conlleva a la formación integral del estudiante en relación a las capacidades de los profesionales que les permitan resolver problemas en un mundo cambiante con responsabilidad social, con espíritu crítico, emprendedor, innovador y creativo. Sin embargo no se debe olvidar que para implementar un programa de cualquier índole requiere contextualizarse y contar con una gestión educativa que prepare la infraestructura y al recurso humano idóneo para lograr resultados de calidad. De ahí la importancia de realizar evaluaciones continúas y sistemáticas que incluyan evaluaciones internas, externas, coevaluaciones $y$ autoevaluaciones para que circunscriban las subjetividades o percepciones de los actores principales del proceso de enseñanza aprendizaje (13).

Por otra parte el escenario que ofrece el mundo postmoderno, con profundas contradicciones entre conocimiento y sabiduría; desarrollo científico-tecnológico y bienestar social; donde parece dominar una civilización incapaz de resolver sus problemas más elementales como la pobreza, marginación, desnutrición y la degradación ambiental Brovetto J (14). Asimismo Bernal asegura que el escenario donde la educación juegan un papel importante para orientar a la sociedad hacia un desarrollo sostenible, donde las instituciones de educación superior deben ser sensibles a los problemas sociales y gestionar los cambios para construir una sociedad más justa y con mejor calidad de vida (15).

Al respecto, desde una perspectiva empírica se conoce que los estudiantes comúnmente mencionan cuando se les pregunta sobre lo aprendido en los 8 ciclos, previos al internado, y refieren expresiones de asombro tales como: "¿Por qué pasé de ciclo?, "no me siento preparado", "debieron ser más estrictos, cuando me evaluaban", "no debí haber aprobado", "aún nos falta mucho".

En este contexto, se plantean las siguientes interrogantes: ¿Será que el Plan Curricular desarrollado, no es adecuado y necesita ser modificado?, ¿Cuáles son las Características de las estrategias de enseñanza aprendizajes? Para dar respuesta a estas interrogantes se plantea el siguiente estudio de investigación cuyo objetivo es determinar la percepción de los egresados sobre el proceso enseñanza aprendizaje según dimensiones: plan curricular estrategias de enseñanza aprendizaje y evaluación en una Universidad Privada.

\section{MATERIALES Y MÉTODOS}

El presente estudio de investigación es descriptivo, transversal, de enfoque cuantitativo, busca responder problemas relacionados con la percepción de los egresados sobre el proceso enseñanza aprendizaje. El estudio se desarrolló en la Escuela Académico Profesional de Enfermería de una universidad privada, institución acreditada según los estándares de SINEACE el 2015. La población está constituida por los egresados de la Escuela de Enfermería del año 2017. Conformada 
por 120 egresados. Los criterios de inclusión que se tomaron en cuenta: egresados que hayan culminado el $\mathrm{X}$ semestre académico y acepten en forma voluntaria, participar en el estudio. Los Criterios de Exclusión fueron: egresados, que no asistan el día de la aplicación del instrumento.

\section{RESULTADOS}

A continuación, se muestran las características de los egresados de enfermería de una universidad privada que participaron en el estudio, todos residen en Lima, Perú. Las mujeres representaron el $85 \%(n=102)$ de la población. Las edades estuvieron comprendidas entre los 20 a 50 años con una edad promedio de 29,7 y desviación estándar de 5,2 años; según grupo de edad de 30 a 34 tuvieron el mayor porcentaje de representación con $40 \%$, seguido del grupo de 25 a 29 años con $32,5 \%$, el grupo de 20 a 24 años con $12,2 \%$ y mayores de 35 años 13,3\%. Por otra parte en relación a la percepción general, del proceso de enseñanza aprendizaje desarrollado en su formación académica (Figura 1), el 15\% tiene una excelente percepción, el $57 \%$ una buena percepción, $26 \%$ Regular y $2 \%$ mala percepción.

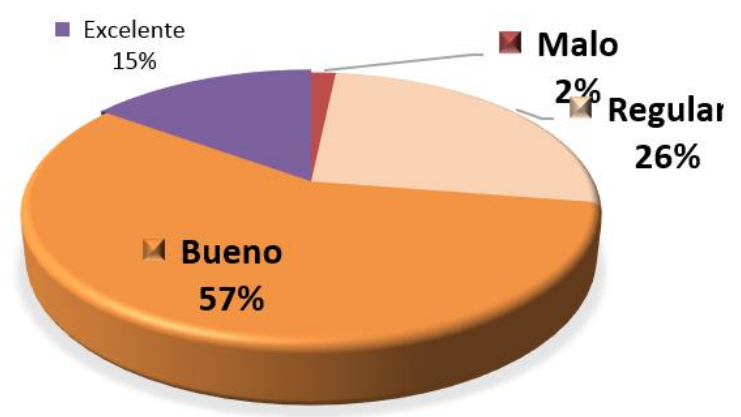

Figura 1. Percepción del proceso enseñanza aprendizaje de los egresados de enfermería en una universidad Privada de Lima $(n=120)$

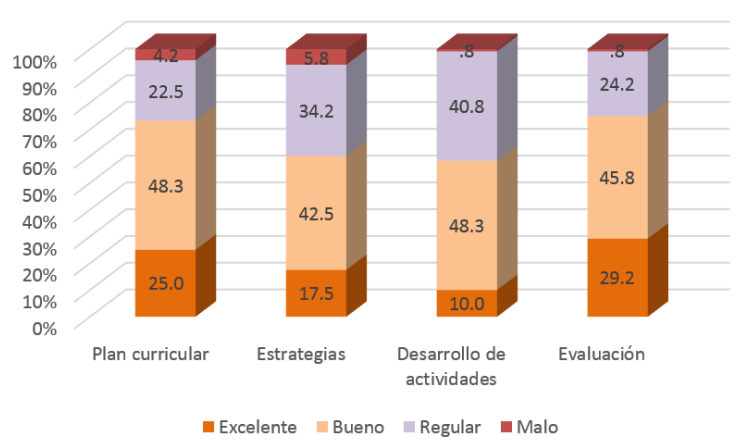

Figura 2. La percepción del proceso enseñanza aprendizaje según dimensiones de los egresados de Enfermería en una universidad Privada de Lima 2018.

\section{DISCUSIÓN}

El perfil considera competencias específicas, clasificadas en cuatro áreas de formación, y un conjunto de competencias generales, propias de la formación integral necesaria para un desempeño profesional de calidad. (Regulación de la Enfermería en América Latina, OPS, RECURSOS HUMANOS PARA LA SALUD, SERIE. Sede OPS - Catalogación en la fuente Organización Panamericana de la Salud. Área de Fortalecimiento de Sistemas de Salud. Unidad de Recursos Humanos para la Salud "Regulación de la Enfermería en América Latina" (16). De manera tal que la estrategia metodológica empleada (taller) hace posible que estas habilidades interactúen y se apoyen mutuamente a fin de desarrollar el pensamiento crítico como parte del proceso intelectual y como producto de sus esfuerzos al interpretar la realidad que lo rodea con todas sus implicaciones, dando prioridad a la razón y honestidad (30). Permitiendo conectar el aprendizaje de los contenidos curriculares con el aprendizaje de los procedimientos para aprender más y mejor esos contenidos.

Asimismo las técnicas de enseñanza aprendizaje son el entramado organizado por el docente a través de las cuales pretende cumplir su objetivo. Son mediaciones que tienen detrás una gran carga simbólica relativa a la historia personal del docente: su propia formación social, sus valores familiares, su lenguaje y su formación académica; también forma al docente su propia experiencia de aprendizaje en el aula. Entre ellas está el socio-drama o simulación, esta técnica consiste en reproducir una situación o problema real. Los participantes deberán representar varios papeles siguiendo instrucciones precisas en un determinado tiempo. La interacción entre los diferentes actores tiene como objetivo encontrar, sobre la marcha, una solución aceptada por las diferentes partes. Asimismo el sistema de evaluación que aplico el docente en el aula fue continuo a través de talleres, trabajos grupales, argumentos, etc., que permitió identificar mis logros y superar mis dificultades en forma oportuna. El 8\% está en desacuerdo y el $92 \%$ de acuerdo de los egresados.

En relación a lo anterior podemos decir que el dinamismo pedagógico (DP) más allá de ser un método de enseñanza es un método de aprendizaje, ya que es dialógico entre los involucrados en el proceso pedagógico, el DP es ambivalente: 1 porque no considera que sea de $A$ o de B, ni tampoco de A para B sino es de A y B, 2 conjuntamente, A y B convergen en un mismo punto: la modificabilidad cognitiva. En estricto 
sentido, el objetivo del DP no es el alumno, ni tampoco el profesor, sino es el aprendizaje en ambos y la autonomía que uno y otro van desarrollando a partir de la modificabilidad cognitiva constante. La modificabilidad cognitiva se va dando en un escenario de ambivalencias complejas, se es positivo y negativo pero también se es ambos. El DP responde no a los contenidos ni a los métodos, sino a las capacidades y valores de los involucrados en el proceso pedagógico (17).

\section{CONCLUSIONES}

Respecto al proceso de enseñanza-aprendizaje, los egresados de la EAP Enfermería de una Universidad Privada de Lima perciben disconformidad respecto al plan curricular, expresado cuando refieren que existe inadecuada secuencia de los cursos y ausencia de algunos considerados importantes como los cursos de carrera. Respecto a las estrategias de enseñanzaaprendizaje y el desarrollo de las actividades, la metodología de las sesiones fue con predominio tradicional haciendo uso solo de multimedia. Evaluación del aprendizaje y acciones de mejora, refieren poca objetividad del proceso de evaluación del aprendizaje, con calificaciones elevadas que no corresponden al nivel real alcanzado por los alumnos, llegando a culminar la carrera sin alcanzar el perfil requerido para afrontar las funciones.

\section{AUTOR DE CORRESPONDENCIA}

Anika Remuzgo Artezano

EP de Enfermería

Universidad Norbert Wiener

Dirección:

Teléfono:

\section{REFERENCIAS BIBLIOGRÁFICAS}

1. Gómez H, Pesántez F. La universidad innovadora. Revista Cubana de Educación Superior (revista en Internet) 2016. (acceso 20 de octubre de 2016); 3 47-63.

2. Castellanos A. V. Estrategia docente para contribuir a la educación de valores en estudiantes universitarios: su concepción e instrumentación en el proceso docente. Revista La educación de valores en el contexto universitario (revista en Internet) 2001 JulioOctubre. (acceso 18 de noviembre 2017); 8 (1).
3. Remuzgo A. Percepción de los alumnos respecto al proceso enseñanza-aprendizaje en el programa de segunda especialización de enfermería en la UNMSM - 2008. (Tesis de Maestria). Lima: Universidad Nacional Mayor de San Marcos.

4. Camere C. "¿Cómo enseñan Psicología los profesores efectivos? Un estudio exploratorio", Perfiles Educativos, tercera época (revista en Internet) 2009 (acceso 20 de noviembre 2017); XXXI (123, pp. 8-26).

5. Peñaloza W. El currículo integral. Ed Tercera. Lima: Centro de Producción Editorial e Imprenta de la Universidad Nacional Mayor de San Marcos, 2005.

6. Peñalosa W. Curriculum Integral. In Curriculum integral (revista en Internet) 2002 (acceso 20 de noviembre); 6 (3).

7. CONEAU. Modelo de Calidad para la Acreditación de la Carrera Profesional Universitaria de Enfermería (Separata especial) 2010 (acceso 20 de noviembre 2017).

8. Miranda E. Evaluación de la Didáctica Aplica durante el desarrollo de la clase magistral por los docentes de la Facultad de Medicina de la UNMSM. Lima: Universidad Nacional Mayor de San Marcos; 1993.

9. Cestari E, Loureiro M. El Proceso De Enseñanza-Aprendizaje En Enfermería. Enfermería Global (revista en Internet) 2005 noviembre (acceso 29 de noviembre 2017); $7(2), 1-7$.

10. Dewey J. Democracy and Education. Edit Macmillan. Co. New York; 1916.

11. Lonergan Bernard. Filosofía de la Educación. Tercera Edición. México: Editorial Universidad Iberoamericana; 1998

12. Woolfolk, Anita Psicología Educativa. 1era edición. México: Editorial Pearson; 2010.

13. Viedma I. Autoevaluación del estudiante en la metodología de ABP. Desarrollo Científico en Enfermería (revista de internet) 2011 Octubre. (acceso 20 de noviembre 2017); 19 (9).

14. BrovettoJ. La Educación Superior y el Futuro. Paris: UNESCO Conferencia mundial sobre la educación superior. La educación superior en el siglo XXI; 5-9 de octubre de 1998.

15. Bernal C. Metodología de la investigación. 3ra edición. Ciudad de México: Pearson Educación; 2010

16. Max Black. Arte, percepción y realidad Red social de literatura, comunidad de lectores Editorial Paidos 1946.

17. Agramonte, A., Sol, D., \& Farres Vázquez, R. Influencia de las estrategias curriculares de la carrera Licenciatura en Enfermería en la calidad del cuidado. Revista Cubana de Enfermería (revista de internet) 2010 (acceso 15 de mayo 2018); 2626(44). 